ज्ञ FRANÇAISE

$\supset$ DE

딜 PEAGOGIE

\section{Revue française de pédagogie}

Recherches en éducation

155 | avril-juin 2006

La motivation scolaire : approches récentes et perspectives pratiques

\title{
Structure de buts, relations enseignants-élèves et adaptation scolaire des élèves : une analyse multi- niveaux
}

Goal structures, student-teacher relationships and students' adaptation to school: a multilevel analysis

Estructura de objetivos, relaciones docentes-alumnos y adaptación escolar de los alumnos: un análisis a varios niveles

Zielstrukturen, Lehrer-Schüler Beziehungen und Schulanpassung der Schüler: eine vielschichtige Analyse

Benoît Galand, Pierre Philippot et Mariane Frenay

\section{(2) OpenEdition \\ Journals}

Édition électronique

URL : http://journals.openedition.org/rfp/225

DOI : $10.4000 /$ rfp.225

ISSN : 2105-2913

Éditeur

ENS Éditions

Édition imprimée

Date de publication : 1 juin 2006

Pagination : $57-72$

ISBN : 978-2-7342-1047-4

ISSN : 0556-7807

\section{Référence électronique}

Benoît Galand, Pierre Philippot et Mariane Frenay, « Structure de buts, relations enseignants-élèves et adaptation scolaire des élèves : une analyse multi-niveaux », Revue française de pédagogie [En ligne], 155 | avril-juin 2006, mis en ligne le 21 septembre 2010, consulté le 19 avril 2019. URL : http:// journals.openedition.org/rfp/225; DOI : 10.4000/rfp.225 


\section{Structure de buts, relations enseignants-élèves et adaptation scolaire des élèves: une analyse multi-niveaux*} Benoît Galand, Pierre Philippot, Mariane Frenay

Cet article cherche à identifier les facteurs qui peuvent influencer la motivation des élèves et plus généralement leur adaptation scolaire. Les recherches passées en revue indiquent que la perception qu'ont les élèves des structures de buts et de la qualité des relations enseignants-élèves sont des éléments importants pour leur engagement scolaire. Une série d'analyses multi-niveaux visant à évaluer si ces perceptions des élèves peuvent refléter des différences de pratiques d'enseignement entre les classes ou si elles reflètent uniquement des interprétations individuelles et/ou des effets de composition sont présentées. Les seuls effets de composition observés de manière récurrente concernent le genre et l'année de scolarité des élèves. Confirmant les résultats antérieurs, les analyses montrent qu'une structure centrée sur la maitrise a un effet positif sur la motivation des élèves. Mais ces analyses montrent en outre qu'une structure centrée sur la performance a un effet négatif sur la motivation des élèves et augmente le risque de victimation. Enfin, ces analyses indiquent que des relations enseignants-élèves de qualité ont un effet pacificateur sur l'agressivité des élèves.

Descripteurs (TEE) : agressivité, conduite de la classe, motivation d'accomplissement, motivation pour les études, rapports maître-élève.

Q u'est-ce qui motive un élève? Pourquoi telle élève s'engage-t-elle avec enthousiasme dans une activité d'apprentissage tandis tel autre élève s'en désintéresse? Plus globalement, pourquoi un élève présente-t-il des conduites qui vont favoriser ses apprentissages et son intégration dans le milieu scolaire (par exemple de la coopération), alors qu'un autre manifeste des réactions qui rendent plus difficiles ses apprentissages scolaires et ses interactions avec autrui (par exemple de l'agressivité) ? Cherchant à dépasser une approche focalisée sur les caractéristiques individuels, les études présentées ci-dessous abordent ces questions sous l'angle des facteurs liés au climat de classe et aux pratiques

\footnotetext{
* La rédaction de cet article a été facilitée par un financement dẹ la Fondation Louvain. Les auteurs remercient particulièrement les élèves qui ont accepté de participer à leurs études.
} 
d'enseignement. Partant du postulat que les apprentissages scolaires demandent à la fois un certain engagement et certains comportements sociaux de la part des élèves (Wentzel, 1999), ces études visent à examiner si certains facteurs peuvent favoriser ou pénaliser l'adaptation scolaire des élèves. Par adaptation scolaire, nous entendons ici l'établissement de transactions relativement constructives entre un élève et son environnement scolaire (1). Nous nous intéresserons d'abord en détail aux facteurs scolaires reliés à la (dé)motivation des élèves, puis nous étendrons notre cadre d'analyse pour nous intéresser à l'effet de certains facteurs scolaires sur les problèmes de violence à l'école.

L'ensemble des propositions théoriques qui s'articulent autour de la théorie des buts (Ames, 1992a; Nicholls, 1989) est une des approches les plus connues dans l'étude de la motivation scolaire. Selon cette approche, les élèves évaluent les événements de leur scolarité et y réagissent en fonction des buts qu'ils poursuivent. La notion de but telle que présentée ici ne se réfère pas à un niveau de performance ou à un objectif précis qu'un élève voudrait atteindre, mais au type de résultats qu'il valorise ou aux raisons qui le pousse à s'engager dans une activité. Selon cette approche théorique, l'important n'est pas seulement de savoir si un élève veut avoir de bonnes notes, par exemple, mais surtout de savoir pourquoi, dans quel but, il veut avoir de bons points. Sans entrer dans les détails, les chercheurs ont identifié deux catégories principales buts poursuivis par les élèves en situation scolaire (Anderman \& Maehr, 1994).

Les buts de maitrise regroupent les buts qui visent l'accroissement de sa compréhension, l'accomplissement de quelque chose que l'on n'a pas réalisé auparavant ou l'amélioration de sa performance. Dans ce type de buts, le processus d'apprentissage, y compris l'effort déployé, est une fin en soi et est valorisé pour lui-même.

Les buts de performance regroupent les buts qui visent l'établissement de la supériorité de sa compétence (par rapport aux autres), l'obtention de jugements de compétence favorables ou l'évitement de jugements négatifs. Dans ce type de buts, un gain en compréhension ou en habilité n'est pas une fin en soi, mais un moyen parmi d'autres d'établir sa supériorité.

Un grand nombre d'études ont examiné les relations entre le type de buts poursuivis par les élèves et une série d'indicateurs de l'engagement scolaire, que ce soit au niveau cognitif, affectif ou comportemental (voir Cosnefroy, 2004). Les résultats de ces études indiquent que les buts de maîtrise sont clairement associés à un engagement soutenu dans la scolarité, tandis que les buts de performance peuvent être associés à des stratégies de défense et de désengagement. Ces recherches indiquent également que ces buts ne sont pas exclusifs l'un de l'autre et les élèves peuvent être motivés conjointement par différents types de buts.

Ces études nous en apprennent beaucoup sur la dynamique motivationnelle des élèves. Elles soulignent notamment que les élèves ne sont pas simplement plus ou moins motivés (quantité de motivation), mais qu'ils peuvent être motivés par des buts différents (qualité de la motivation) et que le type de buts qu'ils poursuivent a des conséquences importantes. Cependant, ces études sont surtout centrées sur les caractéristiques des élèves et les processus individuels. Elles nous renseignent peu sur le rôle que peuvent jouer les agents éducatifs (parents, enseignants, etc.) dans le développement de la motivation scolaire des élèves. L'environnement éducatif dans lequel se trouve un élève a-t-il un impact sur sa dynamique motivationnel ? Certaines pratiques favorisent-elles tel ou tel type de motivation? Ces questions sont bien entendu cruciales pour informer au mieux l'action des personnes qui ont en charge l'éducation et la formation des jeunes. Les études portant sur les déterminants des buts poursuivis par les élèves se divisent en deux catégories: des études expérimentales et des études corrélatives.

\section{LES ÉTUDES EXPÉRIMENTALES SUR LES FACTEURS DE MOTIVATION}

Les études expérimentales consistent à faire varier systématiquement certains paramètres des activités proposées aux élèves, comme la présentation de la tâche ou la forme du feedback. On peut par exemple présenter une tâche comme un test certificatif ou comme un exercice permettant de connaitre et pratiquer différentes compétences. On peut également communiquer les résultats sous forme de commentaires personnalisés ou sous forme de points situant les participants les uns par rapport aux autres. Les élèves sont répartis au hasard dans l'une ou l'autre de ces conditions, et l'on peut comparer leur réaction suivant la situation à laquelle ils ont été confrontés. De nombreux chercheurs ont observé qu'une 
personne tentera spontanément d'améliorer son niveau de compétence ou de compréhension (i) quand elle est confrontée à des tâches qui lui offrent un défi modéré (ni trop facile, ni trop difficile par rapport à son niveau de compétence), (ii) qu'elle n'est pas soumise à un stress physique ou psychologique, et (iii) s'il n'y a pas d'incitants exogènes saillants - récompenses, punitions, points, etc. Autrement dit, ces conditions favoriseraient l'adoption de buts de maîtrise (Nicholls, 1989). Par contre, les gens adopteraient plutôt des buts de performance quand, au lieu de leur être présentée d'une façon « neutre», une tâche qui inclut des habiletés valorisées est présentée (i) comme un test de ces habiletés, (ii) dans un contexte de compétition ou de comparaison interpersonnelle, ou (iii) dans des situations qui augmentent la préoccupation pour l'image que l'on donne de soi (situations à haute visibilité sociale). Ces prédictions ont été largement vérifiées de façon expérimentale, dans différentes tâches et avec des élèves de différents niveaux (Bergin, 1995; Butler, 1993 ; Graham \& Golan, 1991). Par exemple, les résultats obtenus par Butler (1988) indiquent que des élèves ayant reçu des commentaires individuels par rapport à leurs performances antérieures, ont un intérêt et des performances finales supérieures à des élèves ayant reçu des points calculés en fonction de leurs résultats par rapport aux autres ou des points plus des commentaires.

Ces recherches montrent donc que la motivation des élèves est sensible à certains éléments du contexte dans lequel ils sont plongés. Cependant, elles portent sur des tâches très précises, d'une durée limitée, et n'ont pas toutes été réalisées en situation scolaire. Qu'en est-il dans les classes, où les élèves sont confrontés à une grande diversité de tâches, sur de longues périodes, et où de nombreux paramètres interviennent simultanément?

\section{LES ÉTUDES CORRÉLATIVES SUR LES FACTEURS DE MOTIVATION}

En réponse à cette question, des chercheurs se sont intéressés à ce qui se passe dans les écoles. Ils se sont demandés s'il était possible d'identifier des ensembles de pratiques d'enseignement qui mettaient en avant l'atteinte de certains buts, qui valorisaient un certain type de motivation, bref qui instauraient un certain climat motivationnel dans la classe. Leurs travaux mettent en avant, d'une part, des pratiques qui favoriseraient des buts de maîtrise, comme par exemple la mise en place d'activités d'apprentissage qui rencontrent les centres d'intérêt des élèves, le degré d'autonomie accordée aux élèves dans le choix des tâches ou le rythme de travail, l'attention portée à l'effort et aux progrès personnels, la reconnaissance de l'erreur comme partie intégrante de l'apprentissage (Ames 1992a; Meece, 1991). D'autre part, ces travaux identifient des pratiques qui favoriseraient des buts de performance, comme par exemple l'accent mis sur la comparaison entre élèves, la reconnaissance du résultat ou du produit, plutôt que du processus d'apprentissage, la valorisation des élèves les plus performants. Les chercheurs ont baptisé ces ensembles de pratiques d'enseignement «structures de buts » pour souligner qu'il s'agit de pratiques qui favorisent l'adoption de certains buts. En d'autres mots, une structure de buts reflète les buts ou les définitions de la réussite valorisés par les pratiques d'enseignement au sein d'un cours, d'une classe ou d'une école. Comme pour les orientations, les chercheurs ont distingué deux structures de buts (Ames, 1992a) :

- une structure centrée sur la maîtrise, dans laquelle les pratiques pédagogiques valorisent et soutiennent la maitrise, l'amélioration et le développement intellectuel, et où l'accent est donc mis sur le développement des compétences de tous les élèves, quel que soit leur niveau par rapport aux autres ;

- une structure centrée sur la performance, dans laquelle les pratiques pédagogiques favorisent la comparaison sociale et la compétition, et où l'accent est donc mis sur la sélection et promotion des élèves les plus performants.

Quelques études se sont intéressées aux pratiques d'enseignement rapportées par les enseignants. Leurs résultats indiquent que les élèves adoptent davantage de buts de maîtrise dans les classes où les enseignants rapportent des pratiques identifiées comme centrées sur la maîtrise, alors qu'ils adoptent davantage de buts de performance dans les classes où les enseignants rapportent des pratiques identifiées comme centrées sur la performance (Anderman \& Young, 1994; Galand \& Grégoire, 2000). Par ailleurs, la modification de certaines pratiques d'enseignement a des effets repérables sur les orientations de buts des élèves (Nichols \& Miller, 1994 ; Nicholls et al., 1990). Ces quelques résultats soutiennent l'idée que les pratiques d'enseignement affectent le type de motivation des élèves en situation scolaire conformément aux deux types de structures de buts présentées ci-dessus. 
Cependant, la plupart des études disponibles se sont intéressées à la perception qu'ont les élèves des structures de buts dans leur classe ou leur école. Leurs résultats indiquent que la perception d'une structure centrée sur la maîtrise est associée à la poursuite de buts de maîtrise, tandis que la perception d'une structure centrée sur la performance est associée à la poursuite de buts de performance (Anderman \& Anderman, 1999 ; Roeser, Midgley \& Urdan, 1996). En outre, la perception d'une structure centrée sur la maîtrise est liée à l'usage de stratégies de traitement en profondeur des informations à apprendre (Ames \& Archer, 1988), à une diminution des comportements d'évitement (Turner et al., 2002) et à une plus grande satisfaction vis-à-vis de l'école (Treasure, 1997). À l'inverse, la perception d'une structure centrée sur la performance est liée à l'usage de stratégies d'auto-handicape (Midgley \& Urdan, 2001), à la tricherie (Anderman, Griesinger \& Westerfield, 1998), à des états émotionnels négatifs (Galand \& Philippot, 2005) et à des notes scolaires plus faibles (Roeser \& Eccles, 1998). Les résultats de ces études sont très cohérents pour souligner le fait qu'une structure centrée sur la maîtrise est positivement associée à l'engagement scolaire des élèves (Wolters, 2004).

Au-delà des pratiques d'enseignement au sens strict, certains chercheurs se sont dit que des aspects plus sociaux ou relationnels du comportement des enseignants pouvaient également avoir une influence sur la motivation des élèves. Ils se sont donc aussi intéressés à la qualité des relations entre enseignants et élèves, c'est-à-dire au degré de respect, d'équité et de soutien (scolaire et personnel) proposé par les enseignants lors de leurs interactions avec les élèves (Midgley, Feldlaufer \& Eccles, 1989 ; Wentzel, 1997). Plusieurs études montrent que plus un élève perçoit les relations professeursélèves comme étant de bonne qualité, plus il poursuit des buts de maîtrise et plus il est intéressé par sa scolarité (Galand, 2004b ; Roeser et al., 1996 ; Ryan \& Patrick, 2001). Par contre, des relations professeurs-élèves de mauvaise qualité sont associées à l'adoption de buts de performance. De plus, l'existence de relations professeurs-élèves de qualité est négativement liée à la tricherie, à l'agressivité et à l'absentéisme des élèves (Galand \& Dupont, 2002 ; Murdock, Hale \& Weber, 2001).

Un des problèmes qui se pose en travaillant avec les perceptions des élèves est de savoir si les élèves sont motivés parce qu'ils perçoivent un certain climat ou s'ils perçoivent un certain climat parce qu'ils sont motivés. Heureusement, plusieurs des études citées ci-dessus suivent un plan longitudinal (i. e. elles interrogent les mêmes élèves à plusieurs reprises) et indiquent que les perceptions des pratiques éducatives ont bien un effet sur la motivation des élèves, en particulier la perception d'une structure centrée sur la maîtrise (Urdan \& Midgley, 2003). En outre, le passage du primaire au secondaire s'accompagne de changements dans les perceptions, changements qui prédisent l'évolution de la motivation des élèves (Anderman \& Midgley, 1997).

\section{DE L'INDIVIDU À LA CLASSE}

Un autre problème qui se pose néanmoins est de savoir ce que reflètent les perceptions des élèves (Galand \& Philippot, 2005). Ces perceptions reflètent-elles vraiment les pratiques éducatives des enseignants? Ne sont-elles pas fonction des caractéristiques socio-démographiques ou scolaires des élèves? Reflètent-elles réellement des différences entre classes/écoles ou reflètent-elles plutôt des différences individuelles? Ces questions sont fondamentales, car si les perceptions des élèves reflètent des caractéristiques personnelles plutôt que des différences de pratiques d'une classe ou d'une école à l'autre, les résultats présentés ci-dessus ne nous apprennent pas grand chose sur les pratiques pédagogiques à mettre en œuvre pour développer la motivation des élèves. Par contre, si ces perceptions reflètent bien des différences de pratiques, ces résultats nous offrent des pistes d'intervention prometteuses. On pourrait bien sûr penser que le plus simple serait d'interroger directement les enseignants sur leurs pratiques. Les recherches indiquent cependant que les perceptions des élèves sont davantage liées aux observations réalisées par un tiers que les pratiques rapportées par les enseignants eux-mêmes (voir Galand \& Philippot, 2005).

Une manière de traiter ce problème est de procéder à des analyses multi-niveaux (Bressoux, 1994 ; Raudenbush \& Bryk, 2002). Dans ce type d'analyse, on ne considère plus les perceptions des élèves au niveau individuel, mais on en fait la moyenne au niveau de la classe (ou de l'école). De la sorte, on considère les différents élèves d'une classe comme des observateurs des pratiques d'enseignement auxquelles ils sont exposés, la moyenne de leurs observations fournissant une bonne estimation des pratiques effectivement mises en œuvre. La première 
étape de ce genre d'analyse est bien entendu de vérifier les perceptions des pratiques d'enseignement varient effectivement d'une classe à l'autre, autrement dit de vérifier si les réponses des élèves présentent une certaine cohérence en fonction de la classe qu'ils fréquentent. On peut ensuite examiner dans quelle mesure les pratiques d'enseignement $a u$ niveau classe permettent d'expliquer l'engagement individuel des élèves, tout en tenant compte des caractéristiques personnelles des élèves et de la composition de la classe dans laquelle ils se trouvent.

Jusqu'ici, seules quelques études ont utilisé cette méthode d'analyse. Leurs résultats montrent qu'une structure centrée sur la performance au niveau de la classe est liée à un usage accru de stratégies d'auto-handicap par les élèves (Urdan, Midgley \& Anderman, 1998), tandis qu'une structure centrée sur la maîtrise est liée à davantage de demande d'aide (Ryan, Gheen \& Midgley, 1998) et à moins de dépression parmi les élèves (Galand, Philippot \& Lecocq, 2005). Les études de ce type sont cependant rares, n'ont examiné les effets de structures de buts que sur un petit nombre de variables et ne prennent en compte la composition des classes que de manière très limitée. Dans la suite de ce texte, nous allons présenter une série d'études qui ont été menée en Belgique, auprès d'élèves francophones. Ces études ont été ré-analysées au moyen de l'analyse multi-niveaux afin d'évaluer l'effet des structures de buts et de la qualité des relations enseignants-élèves dans différentes classes sur une série d'indicateur de l'adaptation scolaire des élèves. Elles visent également à examiner l'existence d'éventuels effets de composition des classes.

\section{MÉTHODE DE RECHERCHE ET D'ANALYSE}

Dans les études qui suivent, nous avons donc interrogé les élèves sur les pratiques de leurs enseignants reflétant une structure de buts centrée sur la maîtrise, une structure de buts centrée sur la performance, et la qualité des interactions sociales proposées aux élèves. Le questionnaire utilisé à cet effet a fait l'objet d'une validation détaillée (Galand \& Philippot, 2005). Ce questionnaire comprend cinq items portant sur une structure centrée sur la maîtrise (par ex. : " Les professeurs essayent que les élèves puissent travailler à leur propre rythme $"$ ), quatre items portant sur une structure centrée sur la performance (par ex.: "Quand les professeurs posent des questions, c'est surtout aux bons élèves qu'ils demandent de répondre ") et dix items portant sur les relations enseignants-élèves (par ex. : "Les professeurs font leur possible pour aider les élèves $\%$ ). Les réponses se font sur une échelle à sept points allant de «tout à fait faux " à " tout à fait vrai ». Les analyses psychométriques indiquent que les items se regroupent selon les trois échelles prévues et que les réponses sont cohérentes au sein de chaque échelle. Les participants à ces études proviennent des six années et des différentes filières que compte l'enseignement secondaire en Belgique. Ils se répartissent quasi-équitablement entre filles et garçons représentent une grande variété d'origines sociales et culturelles. Des précisions complémentaires concernant les échantillons étudiés figurent en bas des tableaux de résultats.

Notre première démarche d'analyse a été de vérifier si les réponses des élèves d'une même classe présentaient une certaine cohérence et se distinguaient des réponses des élèves des autres classes. Les analyses mutliniveaux indiquent de fait des différences substantielles concernant les pratiques d'enseignement perçues par les élèves d'une classe à l'autre. Dans les différents échantillons étudiés, environ $28 \%$ de la variance dans la perception qu'ont les élèves d'une structure centrée sur la maîtrise se situe entre les classes. Pour la perception d'une structure centrée sur la performance, environ $17 \%$ de la variance se situe entre les classes. Pour la perception de la qualité des relations enseignantsélèves, cette proportion s'élève à environ $30 \%$. Ces résultats indiquent que la perception des pratiques de ses enseignants que rapporte un élève est certes fonction de ses expériences personnelles et de ses interprétations subjectives, mais aussi pour une bonne part de la classe qu'il fréquente.

Nous avons ensuite agrégé les pratiques perçues par les élèves au sein de chaque classe, en calculant la moyenne des perceptions individuelles, de manière à former - pour chaque classe - un indicateur de chacune des deux structures de buts et de la qualité des relations enseignants-élèves. Une question qui se pose est de savoir comment ces trois indicateurs sont reliés entre eux. Notre hypothèse était que les relations enseignants-élèves sont en partie conditionnées par les structures de buts, parce que ces structures reflètent des pratiques d'enseignement qui induisent certains types d'interactions (Johnson, Johnson \& Anderson, 1983; Rusbult \& Van Lange, 1996). Une structure centrée 
sur la performance repose, par définition, sur des situations d'apprentissage compétitives qui impliquent une pression normative et une discrimination entre élèves «performants » et «non performants ". Ces caractéristiques rendent plus probable que les élèves perçoivent leurs enseignants comme une source d'injustice et de stress, et qu'ils instrumentalisent leurs relations avec eux (i.e. essayer de plaire ou d'impressionner l'enseignant sans développer une relation de confiance et de soutien; Nicholls, 1989). D'autre part, une structure centrée sur la maîtrise repose sur des situations d'apprentissage individualisées ou coopératives qui offrent davantage d'opportunités pour la négociation du curriculum et des échéances et pour les contacts personnalisés (Nicholls \& Thorkildsen, 1995). Cette structure est aussi moins menaçante pour la compétence perçue des élèves (les erreurs font partie de l'apprentissage, moins d'importance est donnée à la comparaison sociale normative; Rosenholtz \& Simpson, 1984), et véhicule plutôt des attentes élevées envers chaque élève (Wigfield \& Harold, 1992). Ces éléments favorisent une perception de l'enseignant comme équitable, soutenant et digne de confiance. Réciproquement, il est probable que des comportements inéquitables, rigides et manifestant peu de soutien et d'écoute favorisent la perception d'une structure centrée sur la performance plutôt que sur la maîtrise. Les résultats observés au niveau des classes confirment que la qualité des relations enseignants-élèves est négativement associée à une structure centrée sur la performance $(r(170)=-.75)$ et positivement associée à une structure centrée sur la maîtrise $(r(170)=.80)$, ces deux structures étant elles-même négativement reliées $(r(170)=-.63)$.

L'étape suivante de notre démarche d'analyse visait à tester l'effet des structures de buts et des relations enseignants-élèves dans les classes sur l'adaptation scolaire des élèves. Néanmoins, toutes les années et toutes les filières de l'enseignement secondaire étaient représentées parmi les participants à nos études. De plus, ces derniers provenaient d'origines socio-économiques et culturelles très diversifiées. II était donc crucial pour nos analyses de tenir compte à la fois des caractéristiques personnelles des élèves et de la composition des différentes classes. Ceci afin de se prémunir autant que possible du risque d'identifier erronément un effet des pratiques d'enseignement en lieu et place d'un effet des caractéristiques socio-démographiques des élèves. Au niveau des élèves, nous disposions d'informations individuelles concernant le genre, l'âge, la profession des parents, la nationalité des parents et le nombre de redoublement. Quand on prend simultanément en compte ces différentes caractéristiques (dans des régressions multiples), le genre et l'âge sont les seules à être systématiquement associées à l'adaptation scolaire des élèves (Galand, 2001). Comme l'âge est fortement confondu avec l'année d'étude et que celle-ci est prise en compte au niveau classe, le genre est la seule caractéristique personnelle que nous avons gardée dans les analyses. Au niveau de la classe, nous disposions d'informations concernant l'année d'étude, la filière d'étude, le nombre d'élèves, la proportion filles/garçons, le retard scolaire moyen, la proportion de parents nationaux, le niveau socio-économique moyen. Comme les études à ce niveau sont rares, nous avons d'abord systématiquement inclus toutes ces variables dans nos analyses. Toutefois, comme le suggèrent Raudenbush et Bryk (2002), nous avons ensuite à chaque fois recalculé chaque modèle en n'incluant uniquement les variables ayant un effet significatif. Ce sont les résultats de ces derniers modèles qui sont présentés dans les tableaux.

Les analyses ont été réalisées au moyen du logiciel « HLM 6.02 ». Ce logiciel permet d'analyser plusieurs niveaux d'analyse simultanément. Dans le cas qui nous occupe, un premier système d'équations permet de calculer les relations entre les variables de niveau individuel au sein de chacune des classes, en pondérant pour la taille de la classe. Les différences entre classes font ensuite l'objet d'un second système d'équations prenant cette fois en compte les caractéristiques des classes. Pour toutes les analyses présentées ci-dessous, nous avons procédé en trois temps. Nous avons d'abord évalué dans quelle mesure l'indicateur d'adaptation scolaire retenu variait d'une classe à l'autre (modèle nul). Nous avons ensuite examiné dans quelle mesure ces variations inter-classes pouvaient s'expliquer par les caractéristiques des élèves au niveau individuel et au niveau des classes (modèle incluant les effets de composition). Enfin, nous avons testé le fait de savoir si les structures de buts et les relations enseignants-élèves ajoutaient encore quelque chose à l'explication de ces variations inter-classes (modèle incluant les effets de climat). En l'absence de cadre théorique permettant de formuler des hypothèses précises, nous avons considéré par défaut que les effets de variables au niveau classe étaient identiques pour les filles et pour les garçons (effets fixes). 


\section{CLIMAT MOTIVATIONNEL DE LA CLASSE ET MOTIVATION DES ÉLÈVES}

Nous nous sommes d'abord intéressés à l'effet des structures de buts et des relations enseignantsélèves sur la motivation scolaire des élèves. Comme indicateurs de la motivation, nous avons retenu les buts de maîtrise, les buts de performance (voir cidessus), et les buts d'évitement du travail, qui consiste à chercher à avoir le moins de travail possible, à être débarrassé des tâches scolaires avec un minimum d'effort (Meece, Blumenfeld \& Hoyle, 1988). Nous avons aussi retenu le sentiment d'appartenance à l'école, c'est-à-dire la mesure dans laquelle l'école fréquentée par un élève constitue pour lui une communauté au sein de laquelle il se sent reconnu et dont il valorise le fait d'être membre (Goodnow, 1993). Nombre d'études indiquent que ce sentiment est un prédicteur important de l'engagement scolaire des élèves (Midgley, 2001). Les détails des différentes échelles utilisées pour mesurer ces indicateurs de motivation figurent dans Galand et Philippot (2003) et dans Galand (2004b).

Conformément à la théorie des buts et aux résultats rapportés ci-dessus, nous nous attendions à ce que des pratiques d'enseignement reflétant une structure centrée sur la maîtrise favorisent l'adoption de buts de maîtrise, et que des pratiques reflétant une structure centree sur la performance tavorisent l'adoption de buts de performance. À ce stade, il s'agissait plutôt de répliquer des résultats observés dans d'autres systèmes d'enseignement. II n'existe par contre aucune étude multi-niveaux concernant les buts d'évitement du travail et le sentiment d'appartenance à l'école (Galand \& Philippot, 2000). Notre hypothèse était que des contacts positifs avec des enseignants manifestant respect, soutien et reconnaissance, favoriseraient le développement d'une identité scolaire positive et limiteraient l'évitement du travail (Wentzel, 1999). II en serait de même pour une structure centrée sur la maîtrise, qui permet une certaine autonomie via les occasions de choix et de participation qu'elle offre et qui soutient la motivation intrinsèque des élèves (Voelkl, 1994). Une structure centrée sur la performance risquerait au contraire de fragiliser l'estime de soi des élèves et de favoriser la vision de l'école uniquement comme un moyen vers un objectif extérieur (par ex. un statut ou une rémunération élevée), et par conséquent d'empêcher une identification à l'école et de favoriser l'évitement du travail (Marshall \& Weinstein, 1984 ; Steele \& Aronson, 1995).
Les résultats de nos analyses sont présentés dans le tableau I. Ce tableau, comme ceux qui suivent, est organisé de la manière suivante: pour chaque variable, le coefficient intra-classe est d'abord présenté, ce coefficient exprime la proportion de la variance qui se situe entre les classes (ici $12 \%$ ). L'origine (intercept) est ensuite rapportée. Ce coefficient représente l'estimation de la valeur de la variable prédite pour une fille dans une classe ayant de caractéristiques moyennes (dans ce cas-ci 5,4 sur une échelle de 1 à 7). Suivent les coefficients liés aux effets de genre ou de composition de la classe, tels qu'ils apparaissent dans le modèle final. Ces coefficients représentent le changement dans la variable prédite associée avec un changement d'une unité dans le prédicteur (par exemple, les garçons ont en moyenne un niveau de buts de maîtrise égal à 5,4 moins 0,42 soit environ 5 ). Le pourcentage de la part de variance qui se situe entre les classes et dont rendent compte les effets de genre et de composition est également précisé (ici 18\%). Après quoi, les effets liés aux pratiques d'enseignement sont présentés (par exemple, être dans une classe avec une structure centrée sur la maîtrise un point plus élevée que la moyenne est associé à une augmentation de 0,24 des buts de maîtrise), ainsi que le pourcentage de variance inter-classe dont rendent compte ces effets (ici $37 \%$ ). Finalement, sont rapportés le pourcentage de la variance inter-classe et le pourcentage de la variance totale couverts par l'ensemble des effets significatifs observés.

Les résultats figurant dans le tableau l confirment largement les hypothèses proposées. Ces résultats montrent que le sentiment d'appartenance et les buts scolaires des élèves varient d'une classe à l'autre et que ces variations s'expliquent en partie par le genre et l'année scolaire des élèves: les filles et les élèves plus jeunes rapportent un profil motivationnel plus positif (plus de buts de maîtrise, moins de buts d'évitement et un plus fort sentiment d'appartenance). Mais ils montrent également que ces variations entre classes sont pour une bonne part liées à des variations dans les structures de buts que reflètent les pratiques des enseignants. Une structure centrée sur la maîtrise a un effet positif sur les buts de maîtrise et le sentiment d'appartenance et un effet négatif sur les buts d'évitement du travail. Une structure centrée sur la performance a les effets inverses. Les résultats concernant les buts de performance sont plus étonnant. Pour ces buts, les variations entre classes sont davantage 
Tableau I. - Analyses multi-niveaux de l'effet du climat motivationnel de la classe sur la motivation des élèves

\begin{tabular}{|c|c|c|c|c|}
\hline & $\begin{array}{l}\text { Buts de } \\
\text { maîtrise }\end{array}$ & $\begin{array}{l}\text { Buts de } \\
\text { performance }\end{array}$ & $\begin{array}{c}\text { Buts d'évitement } \\
\text { du travail }\end{array}$ & $\begin{array}{c}\text { Sentiment } \\
\text { d'appartenance }\end{array}$ \\
\hline Coefficient intra-classe & .12 & .06 & .04 & .11 \\
\hline $\begin{array}{l}\text { Origine (étendue : } 1 \text { à } 7 \text { ) } \\
\text { Genre Composition classe } \\
\text { Année } \\
\text { Filière } \\
\text { Nombre d'élèves } \\
\text { Retard scolaire } \\
\text { Proportion filles/garçons } \\
\text { Niveau socio-économique }\end{array}$ & $\begin{array}{c}5,4^{\star \star \star} \\
-0,42^{\star \star \star} \\
-0,11^{\star \star} \\
0,09^{\star}\end{array}$ & $\begin{array}{l}3,35^{\star \star \star} \\
0,29^{\star \star} \\
-0,15^{\star \star}\end{array}$ & $\begin{array}{l}3,21^{\star \star \star} \\
0,73^{\star \star \star} \\
0,09^{\star \star}\end{array}$ & $\begin{array}{l}4,85^{\star \star \star} \\
-0,23^{\star \star} \\
-0,12^{\star \star}\end{array}$ \\
\hline$\%$ variance inter-classes & $18 \%$ & $37 \%$ & $15 \%$ & $10 \%$ \\
\hline $\begin{array}{l}\text { Pratiques d'enseignement } \\
\text { Structure centrée sur la maîtrise } \\
\text { Structure centrée sur la performance } \\
\text { Qualité des relations enseignants-élèves }\end{array}$ & $\begin{array}{l}0,24^{\star *} \\
-0,13^{a} \\
-0,09^{a}\end{array}$ & & $\begin{array}{c}-0,14^{a} \\
0,21^{*}\end{array}$ & $\begin{array}{c}0,31^{\star *} \\
-0,23^{*}\end{array}$ \\
\hline$\%$ variance inter-classes & $37 \%$ & $2 \%$ & $45 \%$ & $49 \%$ \\
\hline$\%$ variance inter-classes & $55 \%$ & $39 \%$ & $60 \%$ & $59 \%$ \\
\hline$\%$ variance totale & $10 \%$ & $3 \%$ & $9 \%$ & $7 \%$ \\
\hline
\end{tabular}

Note. $\mathrm{N}$ élèves $=1121$; âgés de 12 à 21 ans $(54 \%$ de filles). $\mathrm{N}$ classes $=69$; de 6 à 27 participants par classe, provenant de 8 écoles. Genre : $0=$ fille, 1 = garçon. ${ }^{*}=p<.05 ;{ }^{* *}=p<.01 ;{ }^{* * *}=p<.001 ;{ }^{a}=p<.15$ Source : Galand, 2001 ; Galand \& Philippot, 2003.

liées à la composition de celles-ci, seule la qualité des relations enseignants-élèves a un effet négatif marginal.

\section{CLIMAT MOTIVATIONNEL DE LA CLASSE ET VIOLENCE À L'ÉCOLE}

Après avoir démontré l'effet des structures de buts et des relations enseignants-élèves sur la motivation des élèves, nous avons cherché élargir notre champ d'investigation. L'idée centrale de notre raisonnement était que l'effet du climat motivationnel induit par les pratiques d'enseignement ne se limitait pas à la motivation des élèves, mais pouvait affecter d'autres aspects de leur adaptation scolaire, en particulier leurs comportements sociaux (Galand et al., 2000).

Kaplan, Gheen et Midgley (2002) ont examiné l'effet des structures de buts sur l'indiscipline des élèves. Leur hypothèse était qu'une structure centrée sur la maîtrise focalisait l'attention sur les tâches à réaliser et diminuait l'anxiété, ce qui réduirait l'indiscipline, alors qu'une structure centrée sur la performance pouvait diminuer l'investissement dans les tâches scolaires et susciter des réactions défensives, ce qui augmenterait l'indiscipline. Leurs résultats confirment cette hypothèse. Dans la même ligne d'idée, notre hypothèse était que les structures de buts et la qualité des relations-enseignants élèves mettent l'accent sur certaines valeurs ou normes de comportements et suscitent certaines réactions émotionnelles qui rendent plus ou moins probables l'apparition de problèmes de comportement à l'école. Plusieurs recherches indiquent en effet que le niveau de violence peut varier considérablement d'une école à l'autre, sans que ces variations puissent être expliquées par la composition du public d'élèves accueillis dans ces écoles (Debarbieux, Dupuch \& Montoya, 1997 ; Galand et al., 2004). Par ailleurs, d'autres études indiquent que le climat 
scolaire, en particulier le niveau de conflit et l'accent mis sur l'apprentissage, a une incidence sur les problèmes de comportement des élèves (Gottfredson, 2001). Notre hypothèse était donc que le climat motivationnel de la classe avait une incidence sur l'agressivité des élèves. Cette hypothèse a été testée d'une double manière. D'une part, nous avons examiné l'impact du climat motivationnel sur trois précurseurs de l'agression. D'autre part, nous avons examiné l'impact du climat motivationnel sur la victimation des élèves.

Les trois précurseurs de l'agression que nous avons étudiés sont l'adoption de croyances favorables à l'usage de la violence physique, la fréquence des émotions de colère, et les tensions entre groupes d'élèves (voir Galand \& Dupont, 2002; Galand \& Philippot, 2005). Les croyances favorables à l'usage de la violence désignent les représentations d'une personne concernant les bénéfices potentiels et la légitimité du recours à la violence physique. Plusieurs recherches indiquent que ces croyances prédisent les conduites agressives, y compris le port d'arme, et sont caractéristiques des personnes délinquantes (Guerra et al., 1995). La colère, de son côté, est un des prédicteurs les plus puissants de l'agression, c'est l'émotion par excellence qui incite à la violence (Lindsay \& Anderson, 2000). Quant aux tensions entre les groupes d'élèves, ils désignent le degré de ségrégation et de conflit entre des catégories sociales saillantes pour les élèves en contexte scolaire (Galand, 2001). Ces tensions sont associées à l'hostilité envers les autres groupes que le sien (Struch \& Schwartz, 1989). Ces trois éléments nous paraissent très intéressant à prendre en compte dans le cadre des interrogations actuelles à propos de la violence à l'école et de l'éducation multiculturelle (Galand \& Philippot, 1999).

Concernant les croyances favorables à l'usage de la violence et les émotions de colère, notre hypothèse est qu'une structure centrée sur la performance, en favorisant la compétition et en réduisant les opportunités de réussite ou de valorisation au sein de l'école, rend plus probable la perception d'autrui comme un obstacle ou une menace et augmente l'utilité de l'agression comme moyen d'action ou de défense. Ce type de structure favoriserait donc l'adoption de croyances soutenant le recours à l'agression et l'apparition de sentiments de colère (Bushman \& Baumeister, 1998). Une structure centrée sur la maîtrise aurait par contre l'effet inverse. Parallèlement, des relations peu équitables et peu soutenantes entre enseignants et élèves augmentent la probabilité de confrontations et de situations perçues comme injustes, tandis que des interactions positives offrent un environnement plus sécurisant et un modèle de relation constructive valorisant le respect d'autrui. La qualité des relations enseignantsélèves serait donc négativement liée à l'adoption de croyances soutenant le recours à l'agression et à la fréquence des émotions de colère (Mikula, Schere \& Athenstaedt, 1998 ; Walgrave, 1986).

Concernant les tensions intergroupes, notre hypothèse est qu'une structure centrée sur la maîtrise aurait pour effet de diminuer celles-ci. Une telle structure augmente en effet la probabilité de rencontrer plusieurs conditions documentées comme étant favorables à l'instauration de contacts intergroupes positifs : avoir une certaine fréquence, permettre une certaine proximité "émotionnelle", se dérouler sur un mode égalitaire, être encouragés par autrui ou par les normes sociales, impliquer une certaine coopération et aboutir à un résultat constructif (Islam \& Hewstone, 1993; Yzerbyt \& Schadron, 1996). À l'inverse, une structure centrée sur la performance instaure une interdépendance négative entre les élèves et ne favorise pas une norme égalitaire, ce qui rendrait moins probable des contacts intergroupes positifs (Brown, 1995). Parallèlement, l'attention et la disponibilité des enseignants pour les élèves que reflète la qualité des interactions enseignants-élèves se traduisent sans doute une gestion plus active et plus efficace des relations entre élèves, ce qui pourrait avoir des répercussions positives sur les relations entre groupes d'élèves (Kinket \& Verkuyten, 1999). De même, il est probable que l'équité dans les comportements vis-à-vis des élèves réduit la saillance de certaines catégories sociales, promeut une norme égalitaire et prévienne les réactions négatives liées au sentiment d'injustice, ayant par-là un effet préventif par rapport aux tensions intergroupes (Bourhis \& Leyens, 1994).

Les résultats présentés dans le tableau $n^{\circ} \|$ concordent largement avec nos hypothèses. Ils indiquent que les trois indicateurs retenus varient clairement d'une classe à l'autre. Ces variations sont en partie liées à la composition des classes: les filles se disent moins favorables à l'usage de la violence et moins souvent en colère ; le recours à la violence est moins bien accepté dans les classes des années supérieures, où le retard scolaire est faible et le niveau socio-économique élevé; la colère et les tensions intergroupes sont plus présentes dans les filières professionalisantes que dans les filières généralistes. De plus, la qualité des relations proposées 
Tableau II. - Analyses multi-niveaux de l'effet du climat motivationnel de la classe sur la violence des élèves

\begin{tabular}{|c|c|c|c|}
\hline & $\begin{array}{l}\text { Croyances } \\
\text { agression }\end{array}$ & Colère & $\begin{array}{c}\text { Tensions } \\
\text { intergroupes }\end{array}$ \\
\hline Coefficient intra-classe & .21 & .15 & .16 \\
\hline $\begin{array}{l}\text { Origine (étendue : } 1 \text { à } 7 \text { ) } \\
\text { Genre Composition classe } \\
\text { Année } \\
\text { Filière } \\
\text { Nombre d'élèves } \\
\text { Retard scolaire } \\
\text { Proportion filles/garçons } \\
\text { Niveau socio-économique }\end{array}$ & $\begin{array}{l}3,45^{* * *} \\
0,74^{\star * *} \\
-0,16^{* * *} \\
-0,03^{\star *} \\
0,33^{\star} \\
-0,33^{\star *}\end{array}$ & $\begin{array}{l}2,28^{\star \star *} \\
0,27^{\star \star} \\
0,15^{\star \star}\end{array}$ & $3,09^{* * *}$ \\
\hline$\%$ variance inter-classes & $46 \%$ & $44 \%$ & $35 \%$ \\
\hline 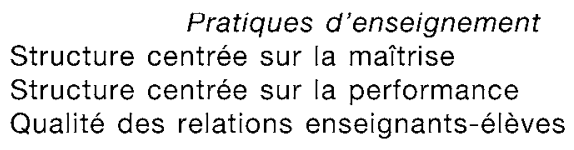 & $-0,47^{\star \star \star}$ & $-0,57^{\star \star \star}$ & $-0,36^{\star \star \star}$ \\
\hline$\%$ variance inter-classes & $33 \%$ & $41 \%$ & $26 \%$ \\
\hline$\%$ variance inter-classes & $79 \%$ & $85 \%$ & $61 \%$ \\
\hline$\%$ variance totale & $25 \%$ & $13 \%$ & $10 \%$ \\
\hline
\end{tabular}

Note. $N$ élèves $=910$; âgés de 12 à 21 ans (53\% de filles). $N$ classes $=55 ;$ de 6 à 27 participants par classe, provenant de 6 écoles. Genre : $0=$ fille, $1=$ garçon. ${ }^{*}=p<.05 ;{ }^{* \star}=p<.01 ;{ }^{* \star *}=p<.001$

Source : Galand, 2001 ; Galand \& Philippot, 1999.

aux élèves par les enseignants a aussi un effet substantiel : meilleure est celle-ci, moins les élèves ont une vision positive du recours à la violence, moins ils se sentent en colère et moins ils perçoivent de tensions intergroupes dans leur école. Les structures de buts ne semblent pas avoir d'effet direct quand la qualité des relations enseignants-élèves est prise en compte.

Une manière complémentaire d'aborder la question du lien entre climat motivationnel de la classe et violence à l'école est de considérer le point de vue des victimes. Il s'agit alors d'interroger les élèves sur les actes dont ils ont été victimes à l'école et d'examiner si la fréquence des ces victimations est fonction des pratiques d'enseignement mise en œuvre dans les classes (Carra \& Sicot, 1997 ; Galand et al., 2004). Nos prédictions sont identiques à celles présentées ci-dessus. Nous nous attendons à ce que les passages à l'acte violent - en tant qu'exutoire à la tension, façon de s'affirmer ou manière d'affaiblir un concurrent - soient plus fréquent dans les classes présentant une structure centrée sur la performance et des relations enseignants-élèves de faible qualité.

Les résultats présentés dans le tableau $\mathrm{n}^{\circ} \mathrm{III}$ sont en accord avec ces prédictions. Ils indiquent que le risque d'être victime de violence à l'école, que ce soit d'atteinte contre ses biens, d'atteinte verbale ou d'atteinte physique, dépend partiellement de la classe fréquentée. Cette variation inter-classes est en partie fonction du genre et de l'année de scolarité: les filles et les élèves des années supérieures sont moins souvent victimes (les atteintes physiques sont aussi moins fréquentes dans les classes avec une plus grande proportion de filles). En outre, une structure centrée sur la performance a également un léger effet sur ces variations entre classes: tous les types d'atteintes sont plus fréquents dans les classes davantage centrées sur la performance. 
Tableau III. - Analyses multi-niveaux de l'effet du climat motivationnel de la classe sur la fréquence de victimation des élèves

\begin{tabular}{|c|c|c|c|}
\hline & $\begin{array}{l}\text { Atteintes contre } \\
\text { les biens }\end{array}$ & $\begin{array}{l}\text { Atteintes } \\
\text { verbales }\end{array}$ & $\begin{array}{l}\text { Atteintes } \\
\text { physiques }\end{array}$ \\
\hline Coefficient intra-classe & .08 & .06 & .07 \\
\hline $\begin{array}{l}\text { Origine } \\
\text { Genre } \\
\text { Année } \\
\text { Filière } \\
\text { Nombre d'élèves } \\
\text { Proportion filles/garçons }\end{array}$ & $\begin{array}{l}0,56^{\star * *} \\
0,16^{\star * *} \\
-0,17^{\star * *}\end{array}$ & $\begin{array}{l}2,29^{* * *} \\
0,27^{\star *} \\
-0,34^{* * *}\end{array}$ & $\begin{array}{l}0,84^{\star \star *} \\
0,27^{\star \star \star} \\
-0,28^{\star * *} \\
0,19^{\star}\end{array}$ \\
\hline$\%$ variance inter-classes & $24 \%$ & $21 \%$ & $50 \%$ \\
\hline $\begin{array}{l}\quad \text { Pratiques d'enseignement } \\
\text { Structure centrée sur la maîtrise } \\
\text { Structure centrée sur la performance } \\
\text { Qualité des relations enseignants-élèves }\end{array}$ & $0,21^{* x}$ & $0,53^{x \times}$ & $0,23^{*}$ \\
\hline$\%$ variance inter-classes & $7 \%$ & $6 \%$ & $4 \%$ \\
\hline$\%$ variance inter-classes & $31 \%$ & $27 \%$ & $54 \%$ \\
\hline$\%$ variance totale & $3 \%$ & $2 \%$ & $5 \%$ \\
\hline
\end{tabular}

Note. $N$ élèves $=2475$; âgés de 12 à 21 ans $(52 \%$ de filles). N classes $=170 ;$ de 5 à 28 participants par classe, provenant de 24 écoles. Genre $0=$ fille, $1=$ garçon. ${ }^{*}=p<.05 ;{ }^{* *}=p<.01 ;{ }^{* * *}=p<.001$

Source : Galand, 2001 ; Galand et al., 2004.

Les résultats figurant dans les tableaux nos II et III pourraient sembler contradictoires, puisque le tableau $n^{\circ}$ II fait ressortir l'effet de la qualité des relations enseignants-élèves, tandis que le tableau $\mathrm{n}^{\circ}$ III souligne l'effet d'une structure centrée sur la performance. Il faut cependant se souvenir que ces deux variables sont fortement corrélées de manière négative et représentent peut-être deux facettes d'un même ensemble. La différence entre les deux tableaux pourrait donc provenir d'un phénomène de colinéarité, et l'interprétation que l'on peut donner des résultats est tout à fait cohérente d'un tableau à l'autre. II semble erroné d'opposer relationnel et pédagogique. Comme indiqué plus haut, il est fort probable qu'il y ait une synergie fonctionnelle entre structures de buts et relations enseignants-élèves (ce qui éclaire également les résultats observés pour les buts de performance, cf. tableau l). II se pourrait néanmoins que les précurseurs de l'agression, d'une part, et les passages à l'acte, d'autre part, soient davantage sensibles à différents types de pratiques, la présence de précurseur n'impliquant fort heureu- sement pas d'office de passage à l'acte. Les précurseurs de l'agression examinés seraient dès lors plus affectés par la qualité des relations enseignants-élèves, alors que les passages à l'acte seraient davantage influencés une structure centrée sur la performance. D'autres recherches seraient nécessaires pour tester cette hypothèse.

\section{DISCUSSION}

Dans cet article, nous avons cherché à identifier les facteurs qui peuvent influencer la motivation des élèves et plus généralement leur adaptation scolaire. Les recherches passées en revue indiquent que la perception qu'ont les élèves des structures de buts et de la qualité des relations enseignants-élèves sont des éléments importants pour leur engagement scolaire. Nous avons ensuite cherché à évaluer au moyen d'analyses multi-niveaux si ces perceptions 
des élèves pouvaient refléter des différences de pratiques d'enseignement entre les classes ou si elles reflétaient uniquement des interprétations individuelles et/ou des effets de composition. Les seuls effets de composition observés de manière récurrente concernent le genre et l'année de scolarité des élèves, ces effets couvrant une part non négligeable des variations inter-classes. Comme dans la plupart des recherches, les filles et les élèves des années supérieures rapportent une adaptation scolaire plus élevée. Les résultats obtenus soutiennent également l'idée qu'au-delà de ces effets de composition, les structures de buts et la qualité des relations enseignants-élèves varient fortement entre les classes et que ces variations sont liées à des différences dans la motivation des élèves, mais aussi à des différences dans leurs comportements sociaux. Confirmant les résultats antérieurs, les analyses présentées montrent qu'une structure centrée sur la maîtrise a un effet positif sur la motivation des élèves. Ces analyses montrent aussi qu'une structure centrée sur la performance a un effet négatif sur la motivation des élèves et augmente le risque de victimation. Enfin, ces analyses indiquent que des relations enseignants-élèves de qualité ont un effet pacificateur sur l'agressivité des élèves.

Ces résultats invitent à recadrer le débat sur les vertus des buts de performance. Ils indiquent en effet que, quels que soient les mérites réels ou supposés des buts performance au niveau individuel (Midgley et al., 2001; Harackiewicz et al., 2002), l'instauration d'une structure de buts centrée sur la performance risque d'avoir des effets délétères sur l'engagement et l'adaptation scolaire des élèves. Autrement dit, si au niveau individuel il apparaît de plus en plus clair que privilégier exclusivement l'un ou l'autre type de buts n'est pas nécessairement plus adaptatif, au niveau des pratiques pédagogiques, il est de plus en plus clair que valoriser les buts de performance n'est pas une bonne stratégie pour soutenir l'engagement des élèves. Les résultats obtenus suggèrent également que la qualité des interactions entre élèves et enseignants peut jouer un rôle dans la prévention des problèmes de violence en milieu scolaire (Gottfredson, 2001). D'où l'importance pour les enseignants d'être attentifs à cet aspect de leur métier, mais aussi d'y être suffisamment formés (Chouinard, 2001).

Plus concrètement, si l'on souhaite soutenir l'adaptation scolaire des élèves, les résultats exposés ci-dessus suggèrent qu'il est utile de mettre en œuvre des pratiques (i) qui offrent aux élèves des opportunités de choix et de participation par rapport au contenu et à la gestion des activités d'apprentissage, (ii) qui aident les élèves à faire le lien entre le contenu des apprentissages et leur vie quotidienne de manière à leur faire découvrir en quoi ces apprentissages leur donnent un pouvoir de compréhension et d'action supplémentaire, (iii) qui véhiculent des attentes positives vis-à-vis des progrès réalisables par chaque élève, et (iv) où l'erreur n'est plus considérée comme une faute mais bien comme un élément à part entière du processus d'apprentissage. D'autre part, vu leurs conséquences négatives, les résultats obtenus invitent à bannir les pratiques ( $v$ ) qui augmentent la saillance ou la valeur accordée à la comparaison sociale entre élèves, (vi) qui véhiculent des attentes négatives vis-à-vis de certains élèves ou (vii) qui accordent la priorité à certains élèves et en négligent d'autres. De plus, les résultats observés suggèrent un impact positif de la disponibilité et de l'écoute des enseignants vis-à-vis des élèves, du soutien qu'ils leur apportent du point de vue scolaire, de l'équité dont ils font preuve dans leurs rapports avec eux, dans les sanctions et dans les évaluations. Par contre, les résultats invitent à éviter tout comportement discriminatoire ou dévalorisant à l'encontre d'un élève (Galand, 2001 \& 2004a).

Si les différents effets décrits ci-dessus rendent compte dans la plupart des cas de la majorite de la variance entre les classes, il reste à chaque fois une part de celle-ci à expliquer. À cet égard, il faut souligner que les échelles utilisées pour mesurer les pratiques d'enseignement ne ciblent que quelques pratiques (voir Galand \& Philippot, 2005). II est tout à fait possible que l'inclusion d'autres dimensions du climat scolaire ou des pratiques d'enseignement permette d'expliquer davantage de variance interclasses (Ames, 1992b; Janosz, Georges \& Parents, 1998). Par ailleurs, la part de variance totale dont rendent compte les structures de buts et les relations enseignants-élèves peut sembler faible. II faut cependant noter qu'il s'agit ici d'effets qui s'appliquent à des classes entières et qui expliquent une portion substantielle des différences d'adaptation scolaire des élèves entre classes. Traduit en fraction d'écart-type, ces effets se révèlent d'amplitude modérée et ont une pertinence pratique certaine. Notre propos n'est pas non plus d'affirmer que la classe est le seul niveau d'analyse pertinent. Les analyses présentées gagneraient sans doute à être étendue au niveau établissement par exemple, ou à d'autres agents de socialisation tels que les parents 
(Frome \& Eccles, 1998). On pourrait également se demander si les effets des structures de buts et des relations enseignants-élèves sont identiques chez tous les élèves. On pourrait faire l'hypothèse que ces effets sont modérés par certaines caractéristiques des élèves, ce qui mériterait d'être exploré plus en détail. Enfin, en terme de perspectives de recherche, il serait intéressant de savoir si les résultats présentés ici sont généralisables à d'autres populations que l'enseignement primaire et secondaire. Certaines études suggèrent que le cadre théorique développé ci-dessus peut s'avérer fécond pour comprendre l'engagement des étudiants de l'enseignement supérieur (Bourgeois, Galand \& Frenay, 2003) et celui des enseignants (Frenay et al., 2003 ; Midgley, 2002).

Benoît Galand benoit.galand@psp.ucl.ac.be

Pierre Philippot pierre.philippot@psp.ucl.ac.be

Mariane Frenay mariane.feray@psp.ucl.ac.be Université catholique de Louvain

\section{NOTE}

(1) Cette définition n'implique pas que toute forme de démotivation ou d'agressivité doivent être considéré comme « anormale » ou pathologique, elle sous-entend par contre que ces comportements risquent d'avoir à terme des conséquences négatives pour les élèves qui les adoptent.

\section{BIBLIOGRAPHIE}

AMES C. \& ARCHER J. (1988). "Achievement goals in the classroom : Students'learning strategies and motivation processes". Journal of Educational Psychology, vol $80, \mathrm{p} 260-267$

AMES C. (1992a). "Classrooms : Goals, structures, and student motivation ". Journal of Educational Psychology, vol. 84 , p. 261-271.

AMES C. (1992b). "Achievement goals, motivational climate, and motivational processes". In G. C. Roberts (éd.) Motivation in sport and exercice. Champaign (USA): Human Kinetics Books.

Anderman E. M. \& Maehr M. L. (1994). « Motivation and schooling in the middle grades ". Review of Educational Research, vol. 64, p. 287-309.

ANDERMAN E. M. \& MIDGLEY C. (1997). "Changes in achievement goal orientations, perceived academic competence, and grades across the transition to middle-level schools". Contemporary Educational Psychology, vol. 22, p. 269-298.

ANDERMAN E. M. \& Young A. J. (1994). «Motivation and strategy use in science: Individual differences and classroom effects". Journal of Research in Science Teaching, vol. 31, p. 811-831.

Anderman E. M.; Griesinger T. \& Westerfield G. (1998). "Motivation and cheating during early adolescence". Journal of Educational Psychology, vol. 90, p. 84-93.

ANDERMAN L. H. \& ANDERMAN E. M. (1999). "Social predictors of changes in students'achievement goal orientations". Contemporary Educational Psychology, vol. 24, p. 2137.
BERGIN D.A. (1995). " Effects of a mastery versus competitive motivation situation on learning ". The Journal of Experimental Education, vol. 63 , p. 303-314.

BOURGEOIS E. ; GALAND B. \& FRENAY M. (2003). " Impact of Students'Perception of the Learning Environment on Self-Regulation in Higher Éducation ". Paper presented at the Annual meeting of the American Educational Research Association: Chicago, April

BouRHIS R. Y. \& LEYENS J.-P. [éd. ] (1994). Stéréotypes, discrimination et relations intergroupes. Liège : Mardaga.

Bressoux P. (1994). "Les effets des écoles sur l'apprentissage de la lecture ». In M. Crahay (éd.), Evaluation et analyse des établissements de formation. Problématique et méthodologie. Bruxelles: De Boeck, p. 123130.

Brown R. (1995). Prejudice: Its social psychology. Cambridge : Blackwell.

Bushman B. J. \& Baumeister R. F. (1998). « Threatened egostism, narcissism, self-esteem, and direct and displaced agression: Does self-love or self-hate lead to violence? ". Journal of Personality and Social Psychology, vol. 75, p. 219-229.

BUTLER R. (1988). "Enhancing and undermining intrinsic motivation : The effects of task-involving and ego-involving evaluation on interest and performance ". British Journal of Educational Psychology, vol. 58, p. 1-14.

ButLER R. (1993). "Effects of task- and ego-achievement goals on information seeking during task engagement ". Journal of Personality and Social Psychology, vol. 65 p. 18-31. 
CARRA C. \& Sicot F. (1997). «Une autre perspective sur les violences scolaires: l'expérience de victimisation". In B. Charlot \& J.-C. Émin (coord.), Violences à l'école: état des savoirs. Paris : A. Colin, p. 61-81.

Chouinard R. (2001). "Les pratiques en gestion de classe: Une affaire de profil personnel et de réflexivité ». Vie pédagogique, $\mathrm{n}^{\circ} 119$, p. 25-27.

Cosnefror L. (2004). "Apprendre, faire mieux que les autres, éviter l'échec: L'influence de l'orientation des buts sur les apprentissages scolaires ". Revue française de pédagogie, $n^{\circ} 147$, p. 107-128.

Debarbieux E. ; Dupuch A. \& Montoya Y. (1997). "Pour en finir avec le "handicap socioviolent ": une approche comparative de la violence en milieu scolaire. In B. Charlot \& J.-C. Émin (coord.), Violences à l'école : état des savoirs. Paris : A. Colin, p. 17-40.

Frenay M. ; Bourgeols E. ; Galand B. ; Wouters P. \& VanderBORGHT C. (2003). "Faculty Involvement in Teaching Tasks within a Changing Curriculum Context: Role of Institutional Supportive Context ». Paper presented at the Annual meeting of the American Educational Research Association : Chicago, april 2005.

FROME P. M. \& ECCLES J. S. (1998). «Parents'influence on children's achievement-related perceptions". Journal of Personality and Social Psychology, vol. 74, $\mathrm{n}^{\circ} 2$, p. $435-452$.

GaLAND B. \& DUPONT E. (2002). «L'impact de la discrimination perçue et de l'intégration scolaire sur l'adoption de croyances favorables à l'usage de la violence physique ". Cahiers internationaux de psychologie sociale, vol. 55 , p. 64-72.

GalAnd B. \& GrÉGoIre J. (2000). "L'impact des pratiques scolaires d'évaluation sur les motivations et le concept de soi des élèves ". L'Orientation scolaire et professionnel/e, vol. $29, n^{\circ} 3$, p. 431-452.

GALAND B. \& PHILIPPOT P. (1999). «Emotional and motivational factors in stereotypisation, inter-groups attitudes and aggressive behaviors in Educational settings". In A. Fisher (chair), Emotional determinant of social behavior. Symposium conducted at XIth general meeting of the European Association of Experimental Social Psychology: Oxford, July 1999.

GalAND B. \& PHILIPPOT P. (2000). The role of school goal structure and teacher-student relationships in work avoidance: A path analysis. In S. Dewitte (chair), Motivational factors that affect student engagement and academic success. Symposium conducted at the 7th Workshop on Achievement and Task Motivation : Louvain, mai 2000.

GalAND B. \& PHILIPPOT P. (2002). «Style motivationnel des élèves du secondaire : Développement d'un instrument de mesure et relations avec d'autres variables pédagogiques". Canadian Journal of Behavioral Science, vol. 34 , p. 261-275.

Galand B. \& Philippot P. (2005). «L'école telle qu'ils la voient: Validation d'une mesure des perceptions du contexte scolaire par les élèves du secondaire ». Canadian Journal of Behavioral Science, vol. 37, p. 138-154.

GaLAND B. (2001). Nature et déterminants des phénomènes de violence en milieu scolaire. Thèse de doctorat: psychologie et sciences de l'éducation, université catholique de Louvain.
GaLAND B. (2004a). «Prévenir les violences à l'école: quelle place pour les pratiques d'enseignement ? ». In M. Frenay \& C. Maroy (éd.), L'école, six ans après le décret "missions": Regards interdisciplinaires sur les politiques scolaires en Communauté française de Belgique. Louvain-la-Neuve: Presses universitaires de Louvain, p. 301-316.

GaLAND B. (2004b). "Le rôle du contexte scolaire et de la démotivation dans l'absentéisme des élèves ". Revue des sciences de l'éducation, vol. 30, $\mathrm{n}^{\circ} 1$, p. 125-142.

Galand B. ; Lecoco C. \& Philiprot P. (2005). « Social relationships at school and students'depression ". In D. Marcotte (Chair), School life and internalizing disorders. Symposium conducted at the annual meeting of the American Educational Research Association : Montréal, avril 2005.

Galand B. ; Philippot P. ; Buidin G. \& LeCOCQ C. (2004). "Violences à l'école en Belgique francophone: Différences entre établissements et évolution temporelle ». Revue française de pédagogie, $n^{\circ} 149$, p. 83-96.

Galand B. ; Philippot P. ; Dupont E. \& Macquet D. (2000). "Violence et démotivation dans les écoles secondaires: Quelles causes pour quelles solutions?». Actes du 1 er Congrès des chercheurs en éducation. Bruxelles: Ministère de la Communauté française de Belgique.

Galand B. ; Philippot P.; Petit S. ; Born M. \& Buidin G. (2004). «Regards croisés sur les phénomènes de violence en milieu scolaire: Elèves et équipes éducatives. Revue des sciences de l'éducation, vol. $30, n^{\circ} 4$, p. 465 486.

Goodnow C. (1993). "Classroom belongin among early adolescent students: Relationships to motivation and achievement ». Journal of Early Adolescence, vol. 13, p. 21-43.

Gottrredson D. C. (2001). Schools and delinquency. Cambridge : Cambridge University Press.

Graham S. \& Golan S. (1991). "Motivational influences on cognition: task involvement, ego involvement, and depth of information processing ". Journal of Educational Psychology, vol. 83, p. 187-194.

Guerra N. G. ; Huesmann R. L. ; Tolan P. H. ; Van Acker R. \& ERON L. D. (1995). "Stressful events and individual beliefs as correlates of economic disadvantage and aggression among urban children $"$. Journal of Consulting and Clinical Psychology, vol. 63, p. 518-528.

Harackiewicz J. M. ; Barron K. E. ; Pintrich P. R. ; Elliot A. J. \& ThrASH T. M. (2002). "Revision of achievement goal theory: Necessary and illuminating ". Journal of Educational Psychology, vol. 94, p. 638-645.

ISLAM M. R. \& Hewstone M. (1993). « Dimensions of contact as predictors of intergroup anxiety, perceived outgroup variability, and out-group attitude: An integrative model ". Personality and Social Psychology Bulletin, vol. 19, p. $700-710$.

Janosz M. ; Georges P. \& PARENT S. (1998). “ L'environnement socio-éducatif à l'école secondaire : Un modèle théorique pour guider l'évaluation du milieu $»$. Revue canadienne de psycho-éducation, vol. 27, p. 285-306.

JoHNSON D. W. ; JOHNSON R. \& ANDERSON D. (1983). « Social interdependance and classroom climate". Journal of Psychology, $\mathrm{n}^{\circ} 114$, p. 135-142. 
Kaplan A. ; Gheen M. \& Midgley C. (2002). " Classroom goal structure and student disruptive behaviour ". Bristish Journal of Educational Psychology, vol. 72, p. 191-211.

KINKET B. \& VERKUYTEN M. (1999). "Intergroup evaluations and social context: A multilevel approach ». European Journal of Social Psychology, vol. 29, p. 219-237.

LINDSAY J. J. \& ANDERSON C. A. (2000). « From antecedent conditions to violent actions: A general affective aggression model ». Personality and Social Psychology Bulletin, vol. 26, p. 533-547.

Marshall H. H. \& Weinstein R. S. (1984). « Classroom factors affecting students'self-evaluation: An interactional model ". Review of Educational Research, vol. 54, p. 301-324.

MefCE J. L. (1991). "The classroom context and students'motivational goals ". In M. L. Maehr \& P. R. Pintrich (éd.), Advances in motivation and achievement. Greenwich : JAl, vol. 7, p. 261-285.

Meece J. L. ; Blumenfeld P. C. \& Hoyle R. H. (1988). « Students'goal orientations and cognitive engagement in classroom activities ". Journal of Educational Psychology, vol. 80 , p. 514-523.

MIDGLEY C. [éd. ] (2001). Goals, goal structures, and patterns of adaptative learning. Mahwah : L. Erlbaum.

Midgley C.; Feldlaufer H. \& ECCles J. S. (1989). «Student/teacher relations and attitudes toward mathematics before and after the transition to junio high school ". Child development, vol. 60, p. 981-992.

Midgley C. ; Kaplan A. \& Middleton M. (2001). «Performance-approach goals: Good for what, for whom, under what circumstances, and at what cost ?". Journal of Educational Psychology, vol. 93, p. 77-86.

Midgley G. ; Kaplan A. ; Middleton M. ; Maehr M. L. ; Urdan T. ; Anderman L. H. ; Anderman E. \& Roeser R. (1998). "The development and validation of scale assessing students'achievement goals orientations". Comptemporary Educational Psychology, vol. 23, p. 113-131.

Mikula G. ; Scherer K. R. \& ATHEnStaedt U. (1998). " The role of injustice in the elicitation of differential emotional reactions ". Personality and Social Psychology Bulletin, vol. 24, p. 769-783.

Murdock T. B. ; Hale N. M. \& Weber M. J. (2001). «Predictors of cheating among early adolescents: Academic and social motivations". Contemporary Educational Psychology, vol. 26, p. 96-115.

Nicholls J. G. \& Thorkildsen T. A. léd. \(1995). Reasons for learning: Expanding the convesation on student-theacher collaboration. New York : Teachers College Press.

Nicholls J. G. (1989). The competitive ethos and democratic Education. Cambridge [Mass.] : Harvard University Press.

Nicholls J. G. ; CobB P. ; WoOd T. ; YACKel E. \& PATAShNick M. (1990). "Assessing students'theories of success in mathematics: Individual and classroom differences Journal for Research in Mathematics Education, vol. 21 , p. 109-122.

Nichols J. D. \& MILLER R. B. (1994). « Cooperative learning and student motivation ". Contemporary Educational Psychology, vol. 19, p. 167-178.
Raudenbush S. W. \& BRYK A. S. (2002). Hierarchical linear models : applications and data analysis methods. London : Sage.

RoEser R. W. \& ECcLes J. S. (1998). “ Adolescents'perceptions of middle school : Relation to longitudinal changes in academic and psychological adjustment $»$. Journal of Research on Adolescence, vol. 8, p. 123-158.

Roeser R. W. ; Midgley C. \& Urdan T. C. (1996). «Perceptions of the school psychological environnement and early adolescents'psychological and behavioral functioning in school: The mediating role of goals and belonging". Journal of Educational Psychology, vol. 88 , p. 408-422.

ROSENHOLTZ S. J. \& SIMPSON C. (1984). "The formation of ability conceptions: developmental trend or socia construction?". Review of Educational Research, vol. $54, n^{\circ} 1$, p. 31-63.

Rusbult C. E. \& Van Lange P. A. M. (1996). «Interdependence processes $"$. In T. E. Higgins \& A. W. Kruglansk (éd.), Social psychology: Handbook of basic principles. New-York: Guilford, p. 564-597.

RYAN A. M. \& PATRICK H. (2001). "The classroom social environment and changes in adolescents'motivation and engagement during middle school ». American Educational Research Journal, vol. 38, p. 437-460.

Ryan A. M. ; Gheen M. H. \& Midgley C. (1998). "Why do some students avoid asking for help ? An examination of the interplay among students'academic efficacy, teachers'social-emotional role, and the classroom goal structure ». Journal of Educational Psychology, vol. 90 p. 528-535.

STEELE C. M. \& ARONSON J. (1995). "Stereotype threat and the intellectual test performance of African Americans ". Journal of Personality and Social Psychology, vol. 69, $n^{\circ} 5$, p. 797-811.

STRUCH N. \& SWARTZ S. H. (1989). "Intergroup agression : Its predictors and distinctness from in-group bias ". Journal of Personality and Social Psychology, vol. 56, p. 364-373.

Treasure D. C. (1997). "Perceptions of motivational climate and elementary school children's cognitive and affective response ". Journal of Sport and Exercise Psychology, vol. 19, p. 278-290.

Turner J. C. ; Midgley C. ; Meyer D. K. ; Gheen M. ; AnderMAN E. M. ; KANG Y \& PATRICK H. (2002). « The classroom environment and students'reports of avoidance strategies in mathematics: A multimethod study ». Journal of Educational Psychology, vol. 94, p. 88-106.

URdan T. \& MidGley C. (2003). « Change in the perceived classroom goal structure and pattern of adaptive learning during early adolescence ". Contemporary Educational Psychology, vol. 28, p. 524-551.

Urdan T. ; Midgley C. \& Anderman E. M. (1998). "The role of classroom goal structure in students'use of self-handicapping strategies". American Educational Research Journal, vol. 35 , p. 101-122.

VOELKL K. E. (1994). « School warmth, student participation, and achievement ». Journal of Experimental Education, vol. $63, n^{\circ} 2$, p. 127-138.

WALGRAVE L. (1986). "La vulnérabilité sociétale: une tentative théorique pour comprendre la délinquance grave et persistante de certains jeunes ». In C. De Troy, 
F. Tulkens \& M. van de Kerchove (éd.), Délinquance des jeunes: politiques et interventions. Bruxelles: StoryScientia.

WENTZEL K. R. (1997). « Student motivation in middle school : The role of perceived pedagogical caring ". Journal of Educational Psychology, vol. 89, p. 411-419.

WENTZEL K. R. (1999). "Social-motivational processes and interpersonal relationships : Impications for understanding motivation at school ". Journal of Educational Psychology, vol. 91, p. 76-97.

WigfiELD A. \& HAROLD R. D. (1992). "Teacher beliefs and children's achievement self-perceptions: A developmental perspective ". In D. H. Schunk \& J. L. Meece (éd.), Student perceptions in the classroom. Hillsdale: L. Erlbaum, p. 95-122.

Wolters C. A. (2004). "Advancing achievement goal theory: Using goal structures and goal orientations to predict students'motivation, cognition, and achievement ". Journal of Educational Psychology, vol. 96, p. $236-250$.

YZeRBYT V. \& SCHADRON G. (1996). Connaître et juger autrui : introduction à la cognition sociale. Grenoble : PUG. 